\section{Pharmacotherapy of neuropathic pain: time to rewrite the rulebook?}
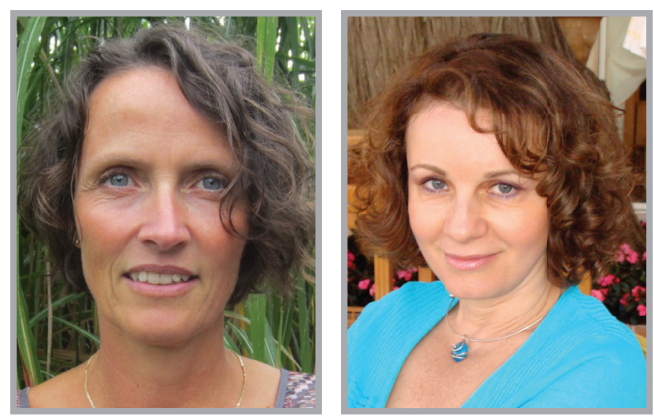

"The International Association for the Study of Pain has declared

2014-2015 the Global Year

Against Neuropathic Pain, which

is an excellent opportunity to

draw attention to the recent

advances in the treatment of

neuropathic pain.”

Nanna B Finnerup ${ }^{*, 1} \&$ Nadine Attal ${ }^{2,3}$

First draft submitted: 24 August 2015; Accepted for publication: 16 September 2015; Published online: 17 December 2015

\section{Neuropathic pain}

Neuropathic pain is pain caused by a lesion or disease of the somatosensory nervous system [1]. Neuropathic pain is a frequent and often disabling condition in patients with cancer or polyneuropathy due to, for example, diabetes, HIV or treatment with chemotherapy, patients with nerve lesions following trauma or surgery and patients with central nervous system lesions such as stroke or spinal cord injury.

\section{Early treatment}

One of the first reports in MEDLINE on how to deal with neuropathic pain dates back to 1843 [2]. This case report describes a woman with neuralgia of the inferior dental nerve. She had excruciating lancinating pain confined to the mental branch of the inferior dental (alveolar) nerve with associated anxiety and fatigue. She had tried various preparations of iron, morphine, leeches and veratria without pain relief. Veratria is an alkaloid obtained from sabadilla seeds causing irritation followed by numbness when applied to the skin, but it may also cause severe electric burning pain, spasms and other symptoms. She was then prescribed strychnine, ioduret of iron and extract of conium. Conium is a homeopathic remedy derived from the hemlock plant, a highly toxic plant used to poison condemned prisoners in ancient Greece - the most famous victim thought to be Socrates. Having continued this treatment for 2 weeks with little improvement, she was prescribed an arsenic solution twice a day with relief of pain after 1 month of treatment. Whether she later developed side effects to the treatment is unknown. In the 1960s, phenytoin and carbamazepine became available, but it was noted that these drugs and alcohol are effective only as long as the sensorium is clouded [3].

\section{Change in treatment}

recommendations

Since these earlier times, the pharmacological treatment of neuropathic pain has continued to be a major challenge. Despite

\section{KEYWORDS}

- neuropathic pain $\bullet$ numbers needed to treat $\bullet$ pharmacotherapy - phenotype $\bullet$ recommendations

- sensory examination

'Department of Clinical Medicine, Danish Pain Research Center, Aarhus University, Denmark

2INSERM U-987, Centre d'Evaluation et de Traitement de la Douleur, Hôpital Ambroise Paré, APHP, Boulogne-Billancourt, France

${ }^{3}$ Université Versailles Saint-Quentin, Versailles, France

*Author for correspondence: Tel.: +45 7846 4230; Fax: +45 78463269; finnerup@clin.au.dk 
"In future clinical trials, stratifying patients according to specific phenotypes may help generate hypotheses for specific predictors of response to specific treatment." the introduction of new and safer drugs and the publication of several randomized controlled trials supporting evidence-based treatment, many patients still do not obtain sufficient pain relief or do not tolerate adequate doses because of side effects.

A first systematic review on the pharmacological treatment of neuropathic pain published in 1999 concluded that only the efficacy of tricyclic antidepressants (TCAs) relied on a sufficiently large total number of patients [4]. Other oral drugs with some evidence for use in neuropathic pain include the sodium channel blockers mexiletine, carbamazepine and lamotrigine, the alpha-2-delta ligand gabapentin, the NMDA antagonist dextromethorphan, opioids and topical capsaicin. In an update from 2005, evidence suggested TCAs as the first-line drug followed by gabapentin and pregabalin as second-line and tramadol and strong opioids as third-line drugs, while results were considered inconsistent for the other previously proposed drugs [5]. In 2010 , despite an increase of $66 \%$ in randomized placebo-controlled trials, it was concluded that there was no evidence for recommending major changes in the treatment algorithm proposed earlier, except with regard to serotonin noradrenaline reuptake inhibitors (SNRIs) that were considered in the algorithm for the first time [6].

\section{NeuPSIG treatment recommendations}

The International Association for the Study of Pain has declared 2014-2015 the Global Year Against Neuropathic Pain, which is an excellent opportunity to draw attention to the recent advances in the treatment of neuropathic pain. The Special Interest Group on Neuropathic Pain (NeuPSIG) of International Association for the Study of Pain recently published a systematic review, a meta-analysis and treatment recommendations using the Grading of Recommendations Assessment, Development and Evaluation [7]. The recommendations for using TCAs, SNRIs, gabapentin (now including gabapentin enacarbil and gabapentin extended release) and pregabalin as first-line drugs were upheld with strong recommendations for use. There were weak recommendations for the use of tramadol and strong opioids, which were recommended as second- and third-line treatments, respectively, when the evidence for desirable and undesirable effects was balanced. This meta-analysis also emphasized the use of several new or recent topical treatments as second- or third-line therapies for peripheral neuropathic pain [7]. These include lidocaine 5\% patches, capsaicin $8 \%$ patches and subcutaneous injections of botulinum toxin type $\mathrm{A}$. These drugs generally have moderate efficacy or low quality of evidence, but their major advantage is that they are applied topically or locally (e.g., subcutaneously) in the painful area and have no or limited systemic side effects.

In comparison with the 2010 review, the 2015 update shows that considerably more trials have used SNRIs, gabapentin and pregabalin. However, the estimated treatment effects for these drugs expressed as the number needed to treat (NNT) have continued to increase, with NNTs ranging from 6.4 to 7.7 in the 2015 review, which means that it is necessary to treat six to eight patients with one of these drugs to obtain one patient with at least $50 \%$ pain reduction when the placebo response is subtracted. The reason for this increase is not completely understood. Interestingly, however, it is paralleled with an increase in placebo responses, which may decrease the assay sensitivity of the trials [8]. Increased quality of study designs, longer trial duration and modifications in the diagnostic algorithms of neuropathic pain might also contribute to such results.

\section{Phenotype-based classification}

These modest NNT results emphasize that the current treatment of neuropathic pain remains a trial-and-error process with few recommendations as to which patients will be likely to respond to which drug. To date, treatment recommendations are based on specific neuropathic pain conditions or on neuropathic pain in general [7,9-10]. By contrast, a mechanism-based classification has been advocated for more than two decades [11-13]. The underlying thought is that the mechanisms responsible for neuropathic pain in individual patients are variable and not directly related to the type of neurological disorder causing the pain. Thus, grouping patients based on shared mechanisms rather than a shared underlying etiology of neuropathy would improve treatment and clinical trials. The first challenge to such a mechanism-based treatment approach is that we do not have reliable and easy tools to assess pain mechanisms in individual patients. Another approach has therefore been to group patients with similar pain phenotypes based on clusters of specific pain descriptors and sensory abnormalities or electrophysiological abnormalities [13]. However, this approach may also be challenging since one symptom may be caused 
by several mechanisms, for example, cold allodynia may be caused by upregulation of the transient receptor potential M8 (TRPM8) on small primary afferents, changes in transient sodium channel conductance, central disinhibition and other mechanisms. Conversely, one mechanism may cause several symptoms. Another challenge to a mechanism-based treatment classification is that some of the drugs that are currently used for neuropathic pain may not necessarily target mechanisms but rather exert a net suppressing effect on the pain pathways.

A further understanding of the link between pain phenotypes and pain mechanisms together with the development of new drugs targeting specific mechanisms may increase trial assay sensitivity and improve future neuropathic pain treatments. In future clinical trials, stratifying patients according to specific phenotypes may help generate hypotheses for specific predictors of response to specific treatments, which may then help design future studies where predictors can be tested a priori. Recently, the effect of oxcarbazepine was found to be better in patients with peripheral neuropathic pain with the socalled irritable nociceptor than those without this phenotype [14]. The irritable nociceptor phenotype was defined by the presence of normal warm and cold detection thresholds (preserved nociceptors) and the presence of allodynia or hyperalgesia to mechanical or thermal stimuli based on quantitative sensory testing. Post hoc analysis suggested that the preservation of nociceptors was more important than the presence of allodynia/hyperalgesia for therapeutic prediction. Further studies are needed to replicate these findings and to better understand the precise sensory phenotype that predicts the response to oxcarbazepine or related drugs. Complete quantitative sensory testing may not always be feasible and an a priori hypothesis of predictors may not be available for all drugs, but the use of specific questionnaires for pain descriptors and bedside tests for sensory changes, for example, loss of thermal or pinprick sensation or touchevoked or cold allodynia, may be a first important step forward.

\section{Financial \& competing interests disclosure}

$N B$ Finnerup receives research funding from the EUROPAIN project funded by the Investigational Medicines Initiative, a public-private partnership between the EU and the European Federation of Pharmaceutical Industries and Associations (EFPIA). N Attal received honoraria from Pfizer, Lilly, Grunenthal, Adir, Astellas, Johnson and Johnson, Sanofi Pasteur Mérieux, Astra Zeneca for participation in clinical trials, advisory boards or speakers bureau. The authors have no other relevant affiliations or financial involvement with any organization or entity with a financial interest in or financial conflict with the subject matter or materials discussed in the manuscript apart from those disclosed.

No writing assistance was utilized in the production of this manuscript.

\section{References}

1 Jensen TS, Baron R, Haanpaa M et al. A new definition of neuropathic pain. Pain 152(10), 2204-2205 (2011).

2 Waters J. Case of neuralgia of the inferior dental nerve. Prov. Med. J Retrosp. Med. Sci. 5(122), 352-353 (1843).

3 Hohmann GW. Psychological aspects of treatment and rehabilitation of the spinal cord injured person. Clin. Orthop. Relat Res. 112, 81-88 (1975).

4 Sindrup SH, Jensen TS. Efficacy of pharmacological treatments of neuropathic pain: an update and effect related to mechanism of drug action. Pain 83(3), 389-400 (1999).

5 Finnerup NB, Otto M, McQuay HJ, Jensen TS, Sindrup SH. Algorithm for neuropathic pain treatment: an evidence based proposal. Pain 118(3), 289-305 (2005).
6 Finnerup NB, Sindrup SH, Jensen TS. The evidence for pharmacological treatment of neuropathic pain. Pain 150(3), 573-581 (2010).

7 Finnerup NB, Attal N, Haroutounian S et al. Pharmacotherapy for neuropathic pain in adults: a systematic review and metaanalysis. Lancet Neurol. 14(2), 162-173 (2015).

8 Dworkin RH, Turk DC, Peirce-Sandner S et al. Considerations for improving assay sensitivity in chronic pain clinical trials: IMMPACT recommendations. Pain 153(6), 1148-1158 (2012).

9 Attal N, Cruccu G, Baron R et al. EFNS guidelines on the pharmacological treatment of neuropathic pain: 2010 revision. Eur. J. Neurol. 17(9), 1113-e88 (2010).

10 Moulin D, Boulanger A, Clark AJ et al. Pharmacological management of chronic neuropathic pain: revised consensus statement from the Canadian Pain Society. Pain Res. Manag. 19(6), 328-335 (2014).

11 Max MB. Towards physiologically based treatment of patients with neuropathic pain. Pain 42(2), 131-137 (1990).

12 Woolf CJ, Bennett GJ, Doherty M et al. Towards a mechanism-based classification of pain? Pain 77(3), 227-229 (1998).

13 Attal N, Bouhassira D, Baron R et al. Assessing symptom profiles in neuropathic pain clinical trials: can it improve outcome? Eur. J. Pain 15(5), 441-443 (2011).

14 Demant DT, Lund K, Vollert J et al. The effect of oxcarbazepine in peripheral neuropathic pain depends on pain phenotype: a randomised, double-blind, placebocontrolled phenotype-stratified study. Pain 155(11), 2263-2273 (2014). 